\title{
Effect of ion bombardment and substrate orientation on structure and properties of titanium nitride films deposited by unbalanced magnetron sputtering
}

\author{
S. Guruvenket and G. Mohan Rao ${ }^{\mathrm{a})}$ \\ Department of Instrumentation, Indian Institute of Science, Bangalore-560 012, India
}

\begin{abstract}
The effect of substrate orientation and ion bombardment during the growth on the structure and properties of TiN films deposited by reactive unbalanced magnetron sputtering has been reported. Films deposited at a nitrogen partial pressure of $5 \times 10^{-5}$ mbar and a current density of 2.50 $\mathrm{mA} \mathrm{cm}{ }^{-2}$ were golden yellow in color, characteristic of stoichiometric TiN. The effect of $\mathrm{Si}(100)$ and $\mathrm{Si}(111)$ substrates on the TiN film along with the substrate bias has been investigated. With an increase in the substrate bias on $\mathrm{Si}(111)$ substrate, $\mathrm{TiN}(111)$ is the most preferred orientation. On a $\mathrm{Si}(100)$ substrate with an increase in the substrate bias, $\mathrm{TiN}(220)$ orientation has been observed. The influence of the substrate on the growth of TiN films has been explained in terms of surface energy. The variation of grain size, resistivity, and the internal stress of TiN films as the function of substrate bias have also been investigated.
\end{abstract}

\section{INTRODUCTION}

Titanium nitride (TiN) has very interesting properties such as high hardness, wear and corrosion resistance, low resistivity, etc. The high hardness value makes it applicable to coat cutting tools, thereby increasing the life of the tool. ${ }^{1,2}$ The lustrous golden yellow color of TiN makes it a good decorative coating. ${ }^{3}$ Low resistivity and diffusion barrier properties of TiN have been used in the microelectronic applications, where it serves as a diffusion barrier between the metallic contact and the semiconducting material. ${ }^{4,5}$ The texture and residual stress in TiN coating influences the performance of the coated part. ${ }^{6}$ TiN film with (111) orientation is considered to have superior resistance to abrasion and wear. ${ }^{7}$

The orientation of a growing film can be tailored by ion assisted growth. Some of the important parameters on which the orientation of the growing film depends are the nature of the substrate (crystalline/amorphous), substrate temperature, ion energy, ion flux, ion density, etc. In a chemical vapor deposition process, the substrate temperature, along with the nature of the substrate, plays a vital role on the orientation of the growing film. ${ }^{8}$ On the other hand, in a physical vapor deposition (PVD) process like magnetron sputtering and ion beam sputtering, it has been observed that along with the substrate nature, the effect of the ion bombardment also plays an important role on the properties of the growing film. Ion bombardment on a growing film influences film properties such as packing density, surface roughness, crystallinity, etc. ${ }^{9}$ Conventionally, a separate ion source is used to focus the ions on the growing film during evaporation techniques. In the case of sputtering, ion bombardment is accomplished to a certain extent by applying a bias to the substrate so that the ions in the glow discharge can be accelerated toward the substrate. However, in this case, the ions are drawn from the parent glow discharge and hence a good control over the

${ }^{a)}$ Electronic mail: gmrao@isu.iisc.ernet.in bombardment of the substrate is difficult. In unbalanced magnetron sputtering, because of the magnetic field configuration, some of the ions from the glow discharge are directed toward the substrate, which can be been utilized to modify the properties of the growing film. ${ }^{10}$ By applying a bias to the substrate, more ions can be accelerated toward the substrate with sufficient energy to assist the growing film. The effect of substrate orientation on the growth and properties of titanium nitride films in magnetron sputter deposition has not been reported to the best of our knowledge, while there are reports on the effect of the ion bombardment on the growing film.

In the present study, we have investigated the effect of substrate and the substrate bias on the growth and properties of TiN films deposited by unbalanced magnetron sputtering. The effect of the ion bombardment on the film properties such as, structure, internal stress, resistivity and grain size were analyzed.

\section{EXPERIMENTAL DETAILS}

The details of the unbalanced magnetron-sputtering cathode have been described elsewhere. ${ }^{11}$ A planar circular titanium disk (99.99\% pure and $100 \mathrm{~mm}$ diameter) has been fixed to the unbalanced magnetron cathode by mechanical clamping. Sputtering was done in $\mathrm{Ar}_{2}+\mathrm{N}_{2}$ mixed gas and the total pressure during sputtering was maintained at $10^{-3}$ mbar. The partial pressure of two gases has been controlled individually. The ultimate pressure obtained in the sputtering chamber is $10^{-5}$ mbar using a combination of diffusion and rotary pump. The distance between the substrate and target distance is $12 \mathrm{~cm}$. Resistivity of the films has been measured using a four-probe technique and the thickness of the films was measured using Sloan profilometer. Crystallinity of the films has been studied by x-ray diffraction using $\mathrm{Cu} K \alpha$ radiation whose resolution is $0.01^{\circ}$. The diffraction 


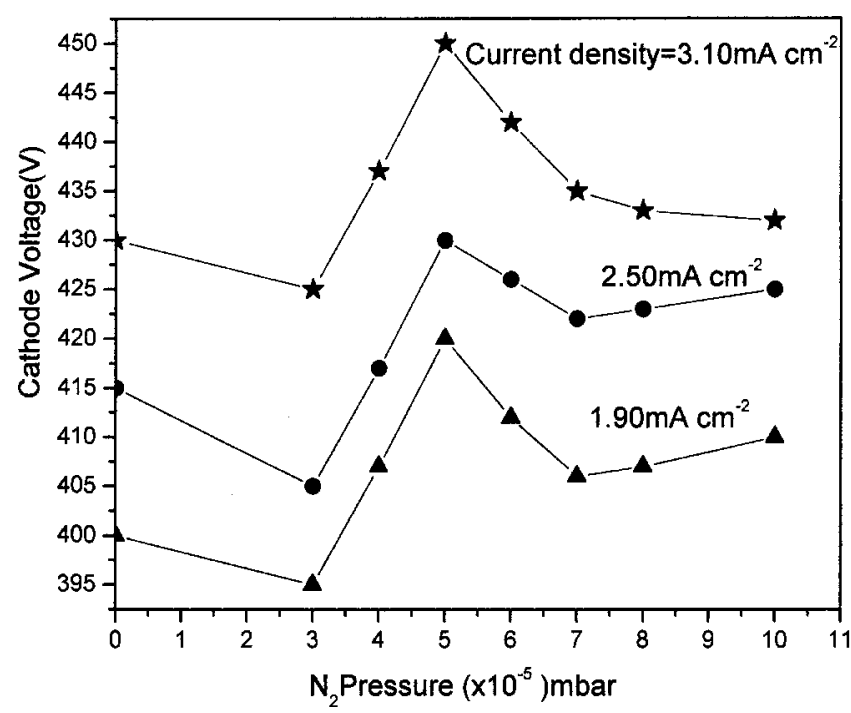

FIG. 1. Variation of the target voltage as a function nitrogen partial pressure.

data has been used to calculate grain size and stress in the films. The internal stress $(\sigma)$ in the deposited film is calculated using

$$
\sigma=-E\left(d_{a}-d_{o}\right) /\left(2 d_{o} Y\right),
$$

where $d_{o}$ and $d_{a}$ are the $d$ spacing of TiN bulk and the $d$ spacing of the film (from an X-ray diffraction experiment), respectively. $E$ and $Y$ are the Young's modulus and the Poisson's ratio of TiN, respectively. ${ }^{7}$ The crystallite size of the TiN film deposited on $\mathrm{Si}(100)$ and $\mathrm{Si}(111)$ were determined using Scherrer's formula, given by $d=\lambda /(\beta \cos \theta)$, where $\lambda$ is the wave length of the $\mathrm{x}$ ray used and $\beta$ is the full width half maximum measured at the $\theta$ value where the peak for the material appears. ${ }^{12}$

\section{A. Optimization of process parameters}

In order to decide the nitrogen partial pressure at which TiN films could be deposited, we have measured the glow discharge characteristics at different nitrogen partial pressures, while maintaining the total pressure constant. The discharge characteristics represent the reactivity between the target ant the reactive gas. Figure 1 shows the variation of the target voltage with the nitrogen partial pressure at three different discharge currents. Figure 1 shows three distinct regions. At low partial pressures of nitrogen, the cathode voltage almost remains constant and starts increasing when the partial pressure is in the region of $3-5 \times 10^{-5}$ mbar. The first region is characteristic of pure metallic target, and the second region is a result of target poisoning effects. At a partial pressure of $3 \times 10^{-5}$, the target starts reacting with the nitrogen gas and TiN formation takes place on the surface. The difference in secondary-electron emission characteristics of $\mathrm{Ti}$ and $\mathrm{TiN}$ results in an increase in the cathode voltage. Similar results have been observed by Petrov et al. ${ }^{13}$ and Tominaga et al. ${ }^{9}$ Though, it is generally assumed that the secondary-electron emission is higher in the case of nitrides than their corresponding metals, ${ }^{14}$ the present results as supported by those reported earlier ${ }^{9,13}$ suggest that in case of
TiN bombarded with argon ions, it is the other way. However, at higher nitrogen pressures, the target poisoning is complete and the cathode voltage shows a decreasing trend. This can be explained to be due to the lower resistivity of TiN (26 $\mu \mathrm{ohm} \mathrm{cm})$ as compared to that of Ti $(45 \mu \mathrm{ohm} \mathrm{cm})$. The critical nitrogen pressure at which target poisoning is complete is determined by the kinetics of the reactive sputtering process, basically the rate of sputtering (determined by the current density on the cathode) and the nitrogen arrival rate on the target (directly related to the nitrogen partial pressure). Consequently, at higher cathode currents, the nitrogen pressure required to deposit stoichiometric TiN films will be higher, for example, in the present case TiN films could be deposited at a nitrogen partial pressure of $7 \times 10^{-5}$ mbar using current density of the order of $3 \times 10 \mathrm{~mA} \mathrm{~cm}^{-2}$, where as at a lower nitrogen partial pressure of $3 \times 10^{-5} \mathrm{mbar}$, the current density is also lower; about $1.90 \mathrm{~mA} \mathrm{~cm}^{-2}$. All further studies reported here have been made on TiN films deposited at a current density of $2.50 \mathrm{~mA} \mathrm{~cm}^{-2}$ and a nitrogen partial pressure of $5 \times 10^{-5}$ mbar, while maintaining total pressure of $1 \times 10^{-3}$ mbar. The substrates (polished silicon with (100) and (111) orientation) were maintained at ambient temperature, without any deliberate heating.

\section{RESULTS AND DISCUSSION}

\section{A. X-ray diffraction studies}

\section{Crystallinity}

The crystallographic orientation of the films deposited by ion assisted reactive sputtering depends upon the deposition parameters such as the ion flux, ion energy, substrate temperature, nature of the substrate (amorphous/crystalline), etc. The effect of ion energy and ion flux on the orientation of TiN films was analyzed in detail and the growth of TiN films with different orientations like (111), (200), and (220) have been reported. The most commonly observed orientation of TiN deposited at low temperature, under low flux/energy ion bombardment is (111). On the other hand, (200) is the preferred orientation at elevated growth temperature. It has been observed that with an increase in the ion/atom ratio, the orientation changes from (111) to (200) while the ion energy and substrate temperature are maintained. ${ }^{15}$ Recently, we observed that the orientation of TiN films deposited by reactive electron cyclotron resonance (ECR) plasma sputtering was (200) irrespective of the nature of the substrate. ${ }^{16}$ Though the ion energy is low in ECR plasma, this was attributed to the higher ion flux, two orders higher than the conventional ion beam process. It was also shown earlier that it is the ion flux rather than the ion energy that influences the formation of even metastable phases like diamond and cubic boron nitride. ${ }^{17,18}$ Thus, the ion flux, ion density, and ion energy seem to play a very important role in inducing crystallization changes in the deposited films. Moderate (200) and (220) texture was found in magnetron-sputtered TiN coatings, as compared to the very predominant (111) texture in the other PVD TiN coatings. ${ }^{19}$ In the present investigation, we studied the change in the preferred orientation of TiN film deposited 

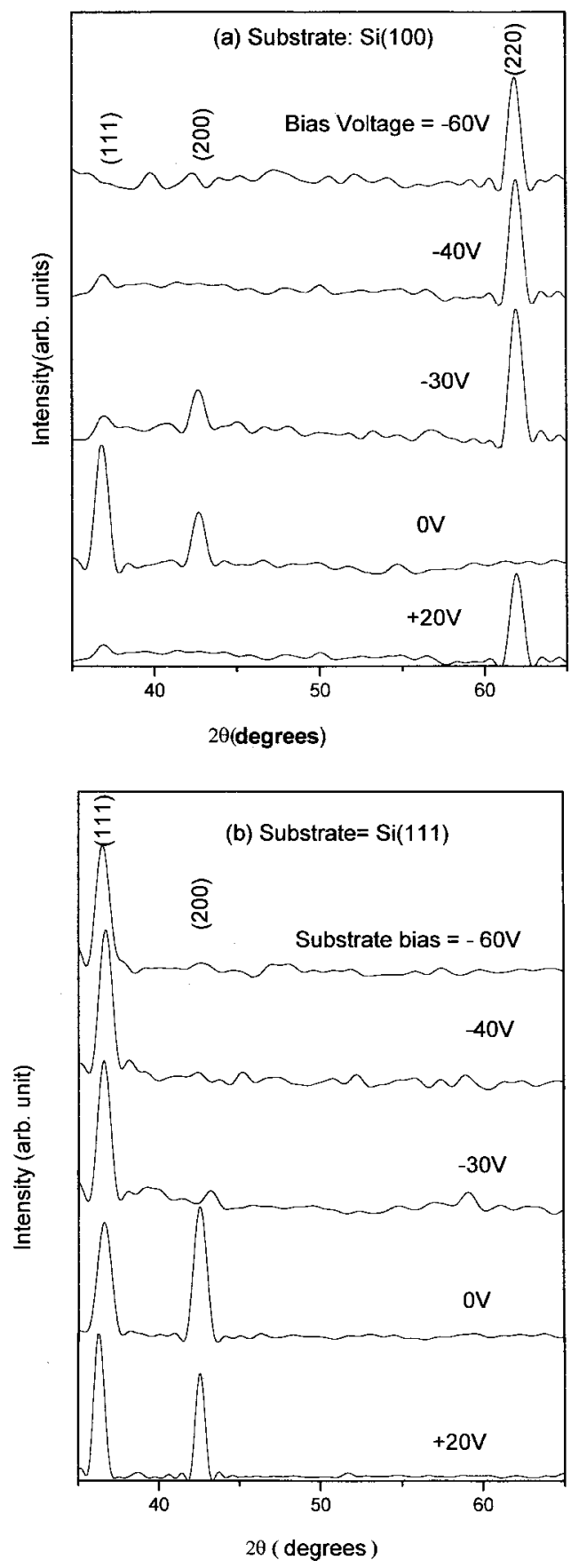

FIG. 2. (a) XRD pattern for TiN films deposited with different substrate bias on a $\mathrm{Si}$ (100) substrate. (b) XRD pattern for TiN films deposited with different substrate bias on a $\mathrm{Si}$ (111) substrate.

on $\mathrm{Si}(100)$ and $\mathrm{Si}(111)$ substrates with the bias voltage which influences the energy of the bombarding ions.

Figure 2(a) shows the variation in the x-ray diffraction (XRD) pattern with substrate bias on $\mathrm{Si}(100)$ substrate. The TiN films deposited without any substrate bias were found to be polycrystalline with (111) and (200) orientation. A positive bias of $+20 \mathrm{~V}$ on the substrate showed a predominantly (220) orientation in the deposited films. When the negative bias of $-30 \mathrm{~V}$ is applied the orientation changes from (111) to (200), and the further increase in the bias voltage shows that the orientation is along the (220) direction. Thus, with the increase in the negative bias, the TiN film is oriented in the (220) direction on a $\mathrm{Si}(100)$ substrate. Figure 2(b) shows the changes in the orientation of TiN film deposited on a $\mathrm{Si}(111)$ substrate with the bias voltage. At $+20 \mathrm{~V}$ bias, the orientation is along (111) and (200) direction and with a decrease in the bias voltage to $0 \mathrm{~V}$, the same trend is observed, but the peak corresponding to (200) phase increases. When the substrate is biased negatively, the orientation along (200) orientation vanishes and only the (111) peak remains.

The change in the orientation of the films shown in Fig. 2 can be explained in terms of the energy $W_{h k l}$ which is the sum of the $S_{h k l}$ the surface energy and the strain energy $U_{h k l}$. Film orientation is significant because both $S_{h k l}$ and $U_{h k l}$ have directional properties. The surface energy of the monoatomic face-centered-cubic (fcc) material is minimum in (111) plane. ${ }^{20}$ Thus, it can be assumed that the surface energy of TiN is also minimal in the (111) plane, even though TiN is not a monoatomic cubic material. Minimization of the surface energy along (111) orientation is considered the driving force for the orientation of TiN along (111) orientation. Zhao et al. ${ }^{21}$ considered that TiN is not monoatomic (fcc), but it is two-component fcc with a NaCl-type structure and thus they calculated the surface energy at different planes, which indicates that the (200) orientation has a minimum surface energy and (111) has a maximum surface energy. The surface energy can also be evaluated by sublimation energy consideration as done by Pelleg et al. ${ }^{22}$ which indicates that the surface energy of the (100) plane is minimum and the (110) is the next followed by (111). It should be noted that the surface energy is independent of film thickness, whereas the strain energy increases linearly with the thickness of the film. Therefore, at lower thickness surface energy term is significant and (100) orientation with a minimum surface energy may expected. At a larger film thickness, the strain energy becomes dominant and (111) and (110) might be expected. Thus, a critical thickness exists above which the strain energy is dominant and below which surface energy becomes dominant. In our present investigation, it has been observed that the thickness of TiN film deposited $(300 \mathrm{~nm})$ is well below the critical thickness reported by Pelleg et al., and hence the surface energy is the driving factor for the oriented growth of the TiN. Thus, according to these results, the most preferred orientation of the TiN must be (100) followed by (110), and is shown in our experimental results.

In this present investigation, we found that the films deposited on $\mathrm{Si}(111)$ substrates find a favorable condition with ion assistance to grow in the direction of the substrate, whereas in case $\mathrm{Si}(100)$ substrates, the same condition leads to (220) orientation. The high ion density in case of ECR plasma was favorable for (200) orientation. ${ }^{16}$ In order to induce (100) orientation growth on a $\mathrm{Si}(100)$ substrate, either a higher substrate temperature or a higher ion flux as shown in on ECR sputtering films may be useful. An earlier report ${ }^{23}$ also suggests oriented (100) growth in TiN films deposited at a higher substrate temperature. It has been reported that by 


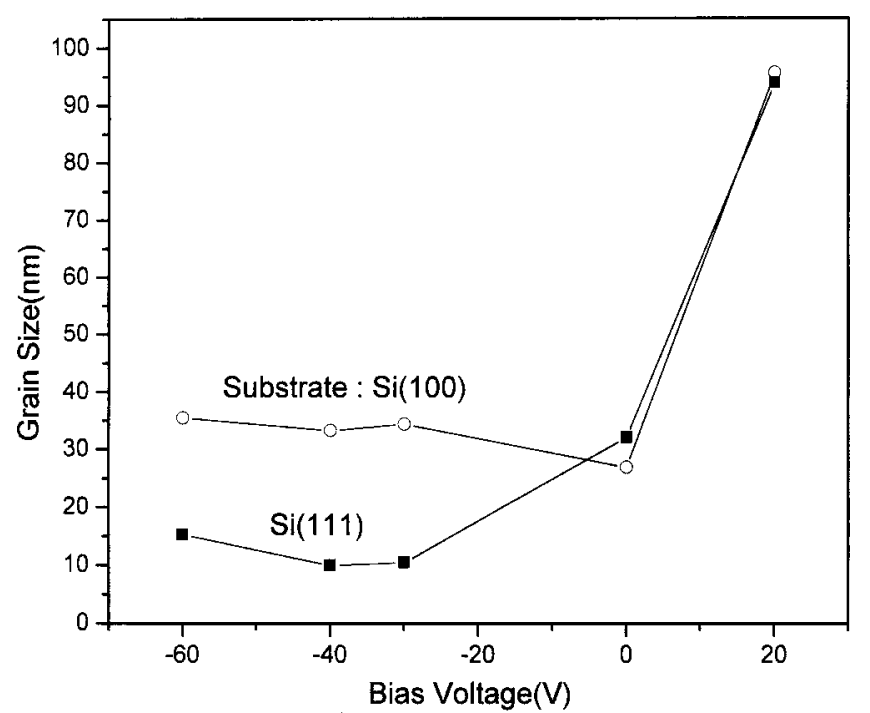

FIG. 3. Grain size variation due to substrate bias.

increasing the ion flux and keeping the ion energy constant, the preferred orientation is along (200) orientation which has a minimum surface energy. ${ }^{15}$ The effects of the substrate bias and the substrate orientation play a dominant role in the preferred orientation along with the deposition parameters such as substrate temperature, ion flux, ion density, etc. With an increase in the substrate bias (ion energy), the change in preferred orientation from (111) to (220) has been reported. ${ }^{21}$ In our case, the ion flux is fixed and with the increase in the substrate bias, the energy of the bombarding ion increases, and hence, ion energy is responsible for the orientation changes of the TiN. The bias voltage (ion energy required), to bring out this change in orientation, is less compared to that reported by Zhao et al. ${ }^{21}$ The reason for this reduction in the bias voltage can be attributed to the unbalanced magnetron geometry, which increases the efficiency of the ionization, and thus a greater number of ions are directed toward the growing film, which in turn helps in the reduction of the bias voltage.

\section{Grain size}

Figure 3 shows the change in the grain size in TiN films deposited on a $\mathrm{Si}(100)$ substrate as a function of the substrate bias voltage. On $\mathrm{Si}(100)$, a maximum grain size of $95 \mathrm{~nm}$ is observed at $+20 \mathrm{~V}$ bias and a minimum grain size of $27 \mathrm{~nm}$ is observed at $0 \mathrm{~V}$ bias condition. An increase in the negative bias increases the grain size and attains almost a constant value $(33 \mathrm{~nm})$ at a bias of $-30 \mathrm{~V}$.

In the case of films deposited on $\mathrm{Si}(111)$ substrates, with a decrease in the bias voltage from +20 to $0 \mathrm{~V}$, the grain size decreases from 94 to $32 \mathrm{~nm}$ and a further decrease in the bias voltage to $-30 \mathrm{~V}$ causes a decrease in the grain size to 10 $\mathrm{nm}$, below which there is no observable change in the grain size. It shows the substrate orientation and the negative bias have no significant contribution on the grain size. However, films grown with positive bias have a higher grain size, prob-

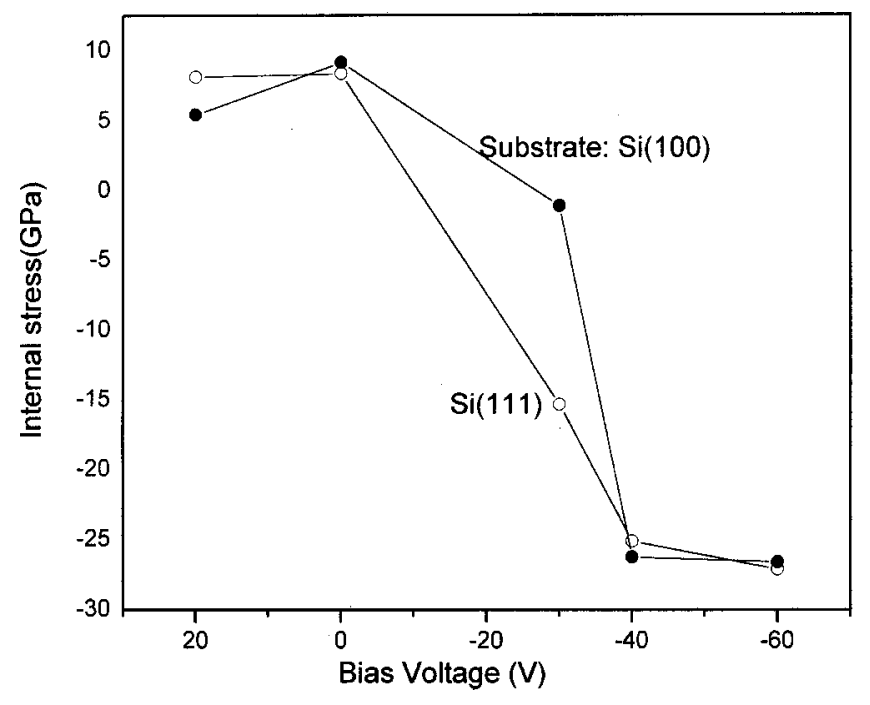

FIG. 4. Stress in TiN films as a function of substrate bias.

ably due to an increase in substrate temperature as a result of electron bombardment. The observed grain size is in accordance with the reported data. ${ }^{23}$

\section{Residual stress}

Ion assisted growth is known to induce stress in the films and the stress is reported to change from compressive to tensile as the energy of the bombarding species is increased. Figure 4 shows the variation in the residual stress in TiN film deposited on $\mathrm{Si}(100)$ substrate with bias voltage. The stress in the deposited TiN film is compressive in nature in positive and zero bias with the value of $5.44 \mathrm{GPa}$ and $9.166 \mathrm{GPa}$, respectively. Films with minimum stress have been grown at a substrate bias of $-10 \mathrm{~V}$. A transformation from compressive to tensile stress takes place with an increase in negative bias. Beyond $-40 \mathrm{~V}$, there is no significant change in the stress and remains at almost a constant value (25 GPa).

In the case of films deposited on a $\mathrm{Si}(111)$ substrate, the behavior is similar to that observed on (100) substrates. Minimum compressive stress of $8.132 \mathrm{GPa}$ is observed at $-20 \mathrm{~V}$ bias. However, the films grown with a substrate bias of $-25 \mathrm{~V}$ were found to have zero stress. Above this value of bias, the films show tensile stress. It can be noticed that when the bias is increased beyond $-40 \mathrm{~V}$, the stress attains almost a constant value (25 GPa).

\section{B. Resistivity}

Figure 5 shows the variation of the resistivity of the TiN film deposited on the $\mathrm{Si}(100)$ substrate at the deposition conditions mentioned earlier. The result indicates that at positive bias, the resistivity of the film is high and gradually decreases with the increase in the negative bias, and beyond $-40 \mathrm{~V}$ again, the resistivity shows an increase. A minimum resistivity of $129 \mu \Omega \mathrm{cm}$ was observed for the films deposited at $-40 \mathrm{~V}$. When the substrate is given a negative bias, the positive ions are attracted toward the substrate and bombard the growing film. Because of this bombardment, the 


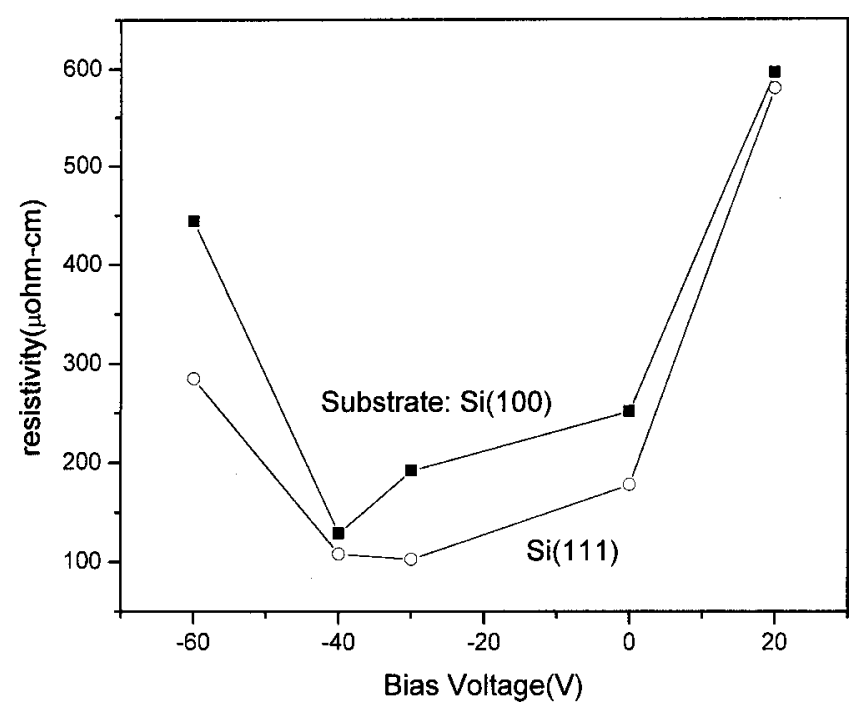

FIG. 5. Resistivity of TiN films as a function of substrate bias.

packing density increases and hence the resistivity decreases. Again, at a higher negative bias, some of the positive ions can get incorporated into the film, which can also increase the resistivity. ${ }^{9}$ We have earlier observed that at a higher negative bias, the nitrogen content in the films increases and this results in an increase in resistivity of TiN films. ${ }^{24}$

Figure 5 also shows the variation in the resistivity of TiN film with the substrate bias deposited on the $\mathrm{Si}(111)$ substrate. Here, a minimum resistivity $(103 \mu \Omega \mathrm{cm})$ was observed at $-30 \mathrm{~V}$ bias. With an increase in the negative bias voltage, the resistivity decreases, and beyond a critical value the resistivity increases. The variation in the bias voltage and the minimum resistivity observed on the two different substrates $[\mathrm{Si}(100)$ and $\mathrm{Si}(111)]$ could account for the different preferred orientation of the film grown on the different substrates as discussed earlier. Patasalas et al. ${ }^{25}$ have obtained a minimum resistivity of $220 \mu \Omega \mathrm{cm}$ at $400{ }^{\circ} \mathrm{C}$ with bias of $-40 \mathrm{~V}$ for the films grown using reactive magnetron sputtering. In our present investigation, we found that even films grown without substrate heating have resistivity of the order of 129 and $103 \mu \Omega \mathrm{cm}$ on $\mathrm{Si}(100)$ and $\mathrm{Si}(111)$ substrates, however with the substrate bias of -30 to $-40 \mathrm{~V}$. Higher ion flux in unbalanced magnetron sputtering ${ }^{11}$ coupled with a substrate bias (which enhances the bombarding energy of the ion) is responsible for the lower resistivity in the present study.

\section{CONCLUSIONS}

The effect of substrate orientation and ion bombardment on the growing TiN films deposited by unbalanced magnetron sputtering was studied. The effect of negative bias (ion energy) on TiN films deposited on $\mathrm{Si}(100)$ substrate shows that the preferred orientation is along (220). On the other hand, the films deposited on a $\mathrm{Si}(111)$ show a preferred orientation along (111) direction. This has been explained by the total energy $W_{h k l}$ which is the sum of surface energy $S_{h k l}$ and strain energy $U_{h k l}$. Compared to earlier studies on bias magnetron-sputtered TiN films, the lower resistivity observed in the present study is attributed to the higher ion flux due to an unbalanced magnetron coupled with enhanced ion energy due to the substrate bias. Internal stress on both the $\mathrm{Si}(100)$ and $\mathrm{Si}(111)$ substrates is compressive in nature for positive bias, and with an increase in the negative bias, the stress becomes tensile and attains almost a constant value beyond $-40 \mathrm{~V}$. It has been observed that the grain size decreases with the increase in the negative bias voltage. A minimum grain size of $10 \mathrm{~nm}$ and $27 \mathrm{~nm}$ was observed on $\mathrm{Si}(100)$ and $\mathrm{Si}(111)$ substrates at $-30 \mathrm{~V}$, respectively. Further increase in the substrate negative bias had no effect on the grain size.

\section{ACKNOWLEDGMENTS}

The authors thank T. Sreeharsha for his help in preparing the coatings.

${ }^{1}$ R. Buhi, H. K. Pulker, and E. Moli, Thin Solid Films 80, 265 (1991).

${ }^{2}$ S. Veprex, J. Vac. Sci. Technol. A 17, 2401 (1999).

${ }^{3}$ E. Valkonen, C.-G. Ribbing, and J.-E. Sundgren, Appl. Opt. 25, 3624 (1986).

${ }^{4}$ P. Engel, G. Schang, and G. K. Wolf, Surf. Coat. Technol. 98, 1002 (1998).

${ }^{5}$ Y. Chiba, T. Omura, and H. Ichimura, J. Mater. Res. 8, 1109 (1998).

${ }^{6}$ J. A. Sue and H. H. Traue, Surf. Coat. Technol. 33, 167 (1987).

${ }^{7}$ A. J. Perry, J. Vac. Sci. Technol. A 8, 1109 (1990).

${ }^{8}$ H.-E-N Cheng and M.-H. Hon, J. Appl. Phys. 79, 8047 (1996).

${ }^{9}$ K. Tominaga, S. Inoue, R. P. Howson, K. Kusaka, and T. Hanabusa, Thin Solid Films 281, 182 (1996).

${ }^{10}$ R. Window and N. Savvides, J. Vac. Sci. Technol. A 4, 453 (1998).

${ }^{11}$ M. Komath, G. Mohan Rao, and S. Mohan, Vacuum 52, 307 (1999).

${ }^{12}$ B. D. Cullity, Elements of X-ray Diffraction (Addision-Wesley, Reading, MA, 1977).

${ }^{13}$ I. Petrov, A. Myres, J. E. Greenead, and J. R. Abeinson, J. Vac. Sci. Technol. A 12, 2846 (1994).

${ }^{14}$ W. D. Westwood, Physics of Thin Films, edited by M. H. Francombe and J. L. Vossen (Academic, New York, 1989), Vol. 14.

${ }^{15}$ L. Hultman, J. E. Sundgren, J. E. Greene, D. B. Bergstrom, and I. Pertrov, J. Appl. Phys. 78, 5395 (1995).

${ }^{16}$ K. Deenamma Vargheese and G. Mohan Rao, Mater. Sci. Eng., B 83, 242 (2001).

${ }^{17}$ N. Savvides, J. Appl. Phys. 74, 3373 (1985).

${ }^{18} \mathrm{~J}$. Amnessen, in Handbook of Plasma processing technology, edited by S. M. Rossnagel, J. J. Cuomo, and W. D. Westwood (Noyes, Park Ridge, New Jersy, 1990), p. 25.

${ }^{19}$ C. D. Quayhaegens, A. M. Jones, J. Haen, and L. M. Stals, Thin Solid Films 258, 170 (1995).

${ }^{20}$ G. Knuyt, C. D. Quayhaegens, J. Haen, and L. M. Stals, Thin Solid Films 258, 159 (1995).

${ }^{21}$ J. P. Zhao, X. Wang, Z. Y. Chen, S. Q. Yang, T. S. Shi, and X. H. Liu, J. Appl. Phys. 30, 5 (1997).

${ }^{22}$ J. Pelleg, L. Z. Zevin, and S. Lungo, Thin Solid Films 197, 117 (1991).

${ }^{23}$ A. R. Phani and J. E. Krazanowski, Appl. Surf. Sci. 174, 123 (2001).

${ }^{24}$ K. Deenamma Vargheese and G. Mohan Rao, Proceedings of the Materials Research Society, 2000, 0119.

${ }^{25}$ P. Patasalas, C. Charitidis, S. Logothetidis, C. A. Dimitriaids, and O. Valassiades, J. Appl. Phys. 86, 5296 (1999). 